\title{
On Statistical Approach to Automated Normal Systolic Blood Pressure Detection in Continuously Monitored Blood Pressure Data
}

\author{
David Kwamena Mensah* Samuel Assabil Francis Eyiah-Bediako \\ Department of Statistics, School of Physical Sciences, University of Cape Coast, Cape Coast, Ghana
}

\begin{abstract}
This paper considers a statistical approach for detecting normal systolic blood pressure pattern from a continuously acquired systolic blood pressure data. Blood pressure monitoring system able to detect subtle changes well in advance in physiological vital signs before clinical emergencies, requires knowledge of the normal blood pressure pattern. Nevertheless, normal data is not always available for pragmatic learning. Ability to learn the normal pattern of systolic blood pressure data is a significant element in the development of robust blood pressure monitoring system. This paper builds on Kernel density approach, based on statistics obtained from novelty scores of the density estimates. The methods are illustrated using simulations and a real data of a continuously acquired systolic blood pressure dataset from Biofourmis Singapore Pte., with detection accuracy of $98 \%$.
\end{abstract}

Keywords: Systolic blood pressure, novelty score, probability density function, vital sign

DOI: $10.7176 / \mathrm{JNSR} / 10-4-05$

Publication date: February $29^{\text {th }} 2020$

\section{Introduction}

As the world is filled with changes so do physiological vital signs. Knowledge of such changes could be informative about the prevention of unforeseen emergencies and to harness important changeovers. Systolic blood pressure (SBP), one of the key components of blood pressure (BP) monitoring, exhibits subtle changes (Evans, Hodgkinson \& Berry, 2001). These changes are a rich source of vital information such as a drift from normal physiology, thus, if discovered and incorporated into model specification, will provide a basic source for building smart automated monitoring systems for the associated vital sign. This will result in the provision of timely interventions, thereby avoiding unforeseen emergencies such as cardiac rest, death, stroke etc. SBP refers to the amount of pressure created in the arteries during the contraction of the heart muscle.

Routine monitoring of systolic blood pressure forms a significant element in the recognition and treatment of subtle changes, indicators of early signs of deterioration in the condition of the heart, etc. (Buist, et al., 1999). As a result, interests in statistical approaches for physiological vital signs monitoring ecosystems have been revived recently and a wealth of research on vital signs modeling has been conducted in the literature.

Conventional techniques adopted for monitoring physiological vital signs, in many hospitals in the World has been the Track-and-Trigger (T and T) system (Centre for Clinical Practice at NICE (UK et al., 2007) and its derivatives such as the modified $\mathrm{T}$ and $\mathrm{T}$ (Gardner-Thorpe, et al., 2006). These systems operate on the principle of scores termed early warning scores (EWS). Manual observations of vital signs of individuals are monitored periodically by nurses with their corresponding EWS computed and compared with their population based normal limits. The inference here is such that if the underlying score or sum of them exceeds a pre-specified threshold derived from the normal limits, an alarm is activated, then the individual is reviewed and care is escalated. Unfortunately, there exists little evidence in support of the use of $\mathrm{T}$ and $\mathrm{T}$ systems (Gao et al., 2007). Also, it is marked with some limitations (Duckitt et al., 2007), with the key one being the use of manual checks by clinical staff. The heuristic nature of thresholds and ranges, the clinical settings from which data are acquired for either validation or the design of the EWS system and the suitability of extrapolating scores obtained from one medical center to another have been criticized in the literature (see e.g., Gao et al., 2007).

Alternative to EWS, is the model-based approach to vital signs monitoring termed the novelty detection. Novelty detection can simply be defined as the identification of data that is deviant based on a given normality model (or some definition of normality) (Khalid, Clifton \& Tarassenko, 2013). An extensive review of this modeling framework can be found in Markou \& Singh (2003a) and Markou \& Singh (2003b). Khalid et al., (2013) developed a Bayesian model averaging approach for automated monitoring of vital signs based on detection of deterioration in manually observed physiological vital signs data. Kernel density approach to vital signs monitoring based on detection of physiological deterioration has been investigated by Pimentel et al., (2013), using vital signs data on the recovery of gastrointestinal post-operative patients. Models of normality were built using vital sign data from normal patients to represent the physiological trajectory of normal recovery.

The performance of novelty detection systems is highly dependent on a correct normal model defined by clinicians or clinical experts. Nevertheless, normal vital signs data may not always be available in some situations. Thus, the question "can statistical methods assist in mining or obtaining physiology specific normal pattern from 
training dataset" has not been addressed in the literature. This paper hypothesizes that statistics derived from the probability density function of training dataset are an attractive source for building automated normal vital signs detector. Thus, a rich source for building statistical procedures which are able to learn from available training data, an appropriate physiology specific normal pattern for contextual and pragmatic detection of subtle changes in physiological vital signs.

The probability density function (pdf) of data provides information on vital features of the data, thus an essential source for the computation of many statistics, for example, the sample mean, sample variance etc. Suppose the expected value of a given random variable is assumed, an appropriate measure of center for such data, then it can be employed as a basis for exploring the contribution of each observation to the center of the distribution of the random variable under consideration. In particular, the corresponding density values of the observations are seen as weights assigned to each observation, thus, can be viewed as their corresponding contributions to the center of the probability distribution of the associated random variable. It is easy to see that an observation spatially close to the center will have a relatively higher contribution than those reasonably far from such center. Also, it is well known that the probability density function underpins several inferential methods, in which varied views are considered for its construction and estimation. For example, in the likelihood approach to inference, it serves as the fundamental pillar. It should be recalled that a statistic is a measurable function of the observations, hence summarizes the data. Statistics obtained based on the probability density function of the data can provide a standard way for data exploration and development of attractive statistical methods for solving scientific problems. This is the direction we propose for addressing the problem of self-learning of normal systolic blood pressure measurements in the context of blood pressure monitoring.

The rest of the paper is organized as follows. Section 2 introduces formally, the statistical methodologies considered. Section 3 provides details on the implementation protocols based on the developed methods. Section 4 discusses in brief, the performance evaluation with some illustrative examples using both real data and simulations outlined and Section 5 concludes.

\section{Methods}

\subsection{Probability density function of data}

Kernel density estimation provides standard approach for both multivariate and single-variate data density estimation from sample data (Pimentel et al., 2013).

The probability density function of a sample data of size, $m$, say, $y$, is estimated based on the model

$$
g\left(y_{i} \mid y, \theta\right)=\frac{1}{m \sqrt{2 \pi \theta^{2}}} \sum_{j=1}^{m} \exp \left(-\frac{\tau_{j}^{2}}{2 \theta^{2}}\right),
$$

where $\tau_{j}=\left(y_{j}-y\right), \theta>0$. Clearly, (1) is a normal mixture model with $m$ components. The components are univariate normal distributions weighted equally with each observation out of the sample acting as the mean. The unknown parameter here is $\theta^{2}$, and is termed smoothing parameter or bandwidth in the context of kernel density estimation. It controls the smoothness of the underlying probability density function.

\subsection{Smoothing parameter estimation}

The choice of smoothing parameter is primal to the performance of kernel density (Bishop, 1994). Thus, the need to adopt a pragmatic approach to its estimation or learning based on empirical data. Bishop (1994) proposed an approach for estimating $\theta$ based on average 10 nearest neighbours in terms of Euclidean distance, for multivariate kernel density estimation. Mensah, Assabil \& Eyiah-Bediako (2019) proposed an empirical scheme for learning suitable smoothing parameter value, $\theta^{2}$, instead of, $\theta$, for univariate density estimation based on weighted distances. Following Mensah et al., (2019), for a single variate, $y$, we consider an estimator, $\hat{\theta}^{2}$ of $\theta^{2}$, based on

$$
\begin{aligned}
\hat{\theta}^{2} & =\sum_{i=1}^{c_{0}+1} \omega_{i} \delta\left(y_{i}, y_{(-i)}\right), i=1, \ldots, m \\
\delta\left(y_{i}, y_{(-i)}\right) & =c_{0}^{-1} \sum_{j=1}^{c_{0}}\left(y_{i}-y_{j}\right)^{a_{0}} \\
\omega_{i} & =\left(\sqrt{\alpha_{0} c_{0} \delta\left(y_{i}, y_{(-i)}\right)}\right)^{-1},
\end{aligned}
$$

where $c_{0}=(m-1), y_{j}=y_{(-i)}$ and we set $a_{0}=2 . \omega_{i}, i=1, \ldots, m$ denotes proximity weighting statistics (proximity weights), and $\alpha_{0}$, an adaptation or tuning parameter that ensures that appropriate smoothing parameter 
value is mined from the data. In other words, it enables contextual learning, making use of the underlying physiological features in order to allow for the estimated value, say, $\hat{\theta}^{2}$, not being too small or too large relative to the data. It should be noted that we have written, $y_{(-i)}$, to mean the remaining data after taking out the ith observation. Mensah et al., (2019) have shown that $\alpha_{0}=0.52$ yields better performance. Following Mensah et al., (2019), we set $\alpha_{0}=0.52$ in all our examples.

\subsection{Normal Systolic blood pressure detector model}

Let $f(y ; \theta)$ denotes the estimated probability density function of a continuously acquired systolic blood pressure dataset, $y=\left(y_{1}, \ldots, y_{m}\right)$, based on model (1). We consider the density-based statistics

$$
T(y)=W(y) y, W(y)=\frac{(S(y)-y)}{\sqrt{V(S(y)-y)}},
$$

where $S(y)=f_{z, \tilde{\mu}}\left(Z(y), \tilde{\mu}_{y}\right) y, Z(y)=-\log (f(y ; \theta)), \tilde{\mu}_{y}$ denotes a robust estimate of the center of the $Z(y)$ statistics, $V(\cdot)$ denotes a variance operator and we have defined

$$
f_{X, Y}(x, y)=x^{-1} y \text {. }
$$

Note that, $T(y)$, is real-valued (i.e. $-\infty<T(y)<\infty)$ and thus, it can be related to physiology in practice. In particular, regarding systolic blood pressure of human, we say, positive values correspond to normal setting since the individual is alive. Concentrating on the positive values, appropriate threshold can be set to learn plausible normal systolic blood pressure measurements for building effective systolic blood pressure monitoring systems.

The rationale for the use of the statistic, $T(y)$, in (5) is due to the asymptotic normality of $W(y)$, justified by the Central Limit Theorem. For sufficiently large dataset of size, say, $m, W(y) \sim N(0,1)$. Thus, it can be utilized to assess values of $y$, which are likely to be normal. The set of values which are highly likely will obviously cluster around the zero line (i.e. within -1 and 1, say). Thus, systolic blood pressure measurements which are highly likely to be normal in terms of a given physiology are located in the high density regions of the empirical density function. Thus, will cluster around regions of low $Z(y)$ features when considered in such feature space. Similarly, deteriorating or abnormal or suboptimal systolic blood pressure values will be located within low density regions of the density function and thus, will generate high $Z(y)$ feature values. When translated into the statistic, $W(y)$, normal values of systolic blood pressure would cluster around the 0 . By this, it is obvious that the statistic, $T(y)$, is informative about the nature of the systolic blood pressure values under its underlying probability density function. As a result, the use of the statistic, $T(y)$, can serve as an important element in developing a principled approach for learning from data, an appropriate normal systolic blood pressure settings in situations where the normal data is not available.

\section{Implementation}

With the high likelihood for the probability density function of real systolic blood pressure to be skewed naturally, it is vital to apply the proposed methods to an appropriate subset of the data. From our experimentation, we noticed that direct application of the proposed method to the training dataset without considering the nature of the density affects the performance. As a result, we outline two different implementation schemes in comparison with the direct method, which we have termed the direct probability density (DPDF) approach. With this implementation, no appropriate data is mined from the given training dataset for further processing. However, model (1) is directly fitted using the training data set, $y$, followed by the application of the statistics in (5), on the corresponding fitted results. The two variant implementation schemes are discussed in brief next.

\subsection{Single-fit probability density (SFPDF) based approach}

With this implementation scheme, the probability density function of the training data is applied once. The process can be detailed as follows:

(1) From the training data, $y$, an appropriate data, $y^{*}$, are derived as

$$
y^{*}=\{y>\tilde{\mu}\} \text {, }
$$


where $\tilde{\mu}$ is the median of $y$

(2) Fit model (1) to $y^{*}$

(3) Apply equation (5) to the results in (2)

\subsection{Double-fit probability density (DFDPF) based approach}

Under this scheme, the estimated probability density function of the data is used twice. First, it is employed as a preprocessing tool for exploiting a region of appropriate data for further processing, using density-based robust statistics. Second, the appropriate data are then subjected to further analysis. The flow of the scheme can be summarized in the following steps.

(1) Fit model (1) to training data, $y$.

(2) Compute the statistics

$$
v\left(y_{i}\right)=y_{i}^{-1} \sum_{j=1}^{N} f\left(y_{i} \mid y_{j}, \theta\right) \text { and } \tilde{\mu}=\operatorname{median}[v(y)],
$$

where $v(y)=\left(v\left(y_{1}\right), \ldots, v\left(y_{N}\right)\right), y_{j}=y_{(-i)}$.

(3) Derive appropriate data, $y^{*}$, from $y$ as $y^{*}=\{y<\tilde{\mu}\}$.

(4) Fit model (1) to $y^{*}$ and apply equation (5)

\section{Performance Assessment}

This section focuses on the implementation and performance assessment of the proposed methods for performing automated normal systolic blood pressure learning. We consider three examples covering real physiological vital signs data example and two simulations based on random perturbation of the real data.

In all our examples, we used the natural threshold informed by the test statistic, $T(y)$, for the detection of normal systolic blood pressure observations. In particular, the algorithm declares an observation as normal if its corresponding test statistic value is positive. First, we introduce in brief the performance measures considered for the evaluation here, before applying them to our examples. The assessment is based on statistical measures of performance utilized for assessing binary classifiers, namely, sensitivity, specificity and accuracy. These measures are usually, computed for binary classifiers based on contingency table shown in Table 1.

\begin{tabular}{lll}
\hline & \multicolumn{2}{c}{ True situation } \\
\cline { 2 - 3 } Predicted situation & Positive & Negative \\
\hline Positive & True Positive $(T P)$ & False Positive $(F P)$ \\
Negative & False Negative $(F N)$ & True Negative $(T N)$ \\
\hline
\end{tabular}

\section{TABLE I: A $2 \times 2$ contingency table for a generic binary classifier}

Sensitivity (true positive rate) measures the proportion of the actual positives that are correctly identified as such by the algorithm. The specificity (true negative rate) of an algorithm measures the proportion of the actual negatives that are correctly identified as such. Based on Table I, sensitivity (SS), specificity (SP) and accuracy (ACC) can be expressed as

$$
\begin{aligned}
S S & =\frac{N(T P)}{N(T P)+N(F N)} \\
S P & =\frac{N(T N)}{N(T N)+N(F P)}, \\
\text { and } & \\
A C C & =\frac{N(T P)+N(T N)}{N(T P)+N(T N)+N(F N)+N(F P)},
\end{aligned}
$$

where $N(Z)$ denotes number of events $Z$. It is clear from (6) and (7) that sensitivity and specificity are 
based on only half of the information in the contingency table. Thus, do not capture all aspects of the performance. From (8), it is apparent that accuracy addresses this by making use of four numbers. Accuracy is therefore more balanced, representative and apprehensive than sensitivity and specificity (Vihinen, 2012). The measure of overall performance of the methods will be based on accuracy.

We consider a two-level assessment for the performance evaluation. First, a true systolic blood pressure annotation was performed with the help of a medical practitioner, based on the current standard physiological settings. Second, the annotation by the developed algorithms (schemes) are compared with the true annotation based on the performance measures discussed above.

\subsection{Example 1: Real Systolic blood pressure data application}

This application is based on real physiological vital signs data collected by the Singapore Heart Foundation for Biofourmis Private Company Limited, Singapore. The dataset is a de-identified data of size, $845 \times 5$, covering Systolic blood pressure (SBP), Diastolic blood pressure (DBP), Mean arterial pressure (MAP), Pulse Rate (PP) and Heart Rate (HR). The data are obtained from adult Asians in the age group, 25 - 62 years, enrolled in a SingHeart study. The subjects were continuously monitored over a period based on varied physiological states, for example, when the individual was sleeping, walking, exercising, sitting etc. We applied the proposed schemes on the SBP, denoted as, $y$. Figure 1 shows plot of the data and its distribution in terms of boxplot. The green lines denote the current convectional normal systolic blood pressure range $\left(\begin{array}{lllll}120 & - & 139 & \mathrm{mmHg}\end{array}\right)$ for adults. The varied physiological states characterizing the dataset are clearly evident with its distribution exhibiting right skewness. Also, there seems to be the existence of outlying observations. The skewness may be due to the potential outliers, which may have been engineered by some factors such as, activity levels, diet, location, etc. Learning the normal pattern or application of detection methods without consideration for potential outliers may lead to poor performance and consequently misleading conclusions.

First, we examine the applicability of the methods to mine training dataset for an appropriate subset for further processing. Figure 2 shows a plot of $V(y)$ against $y$ (left), boxplot of $y^{*}$, the subset data, obtained from the training dataset using SFPDF and DFPDF. The red line is the median, $\tilde{\mu}$, of the $V(y)$ values. Systolic blood pressure values below the red line constitute a subset of the training dataset, $y^{*}$, deemed appropriate for further learning. The distribution of such subset data is shown in terms of boxplot. It is clear that both SFDPF and DFPDF yield the same $y^{*}$.
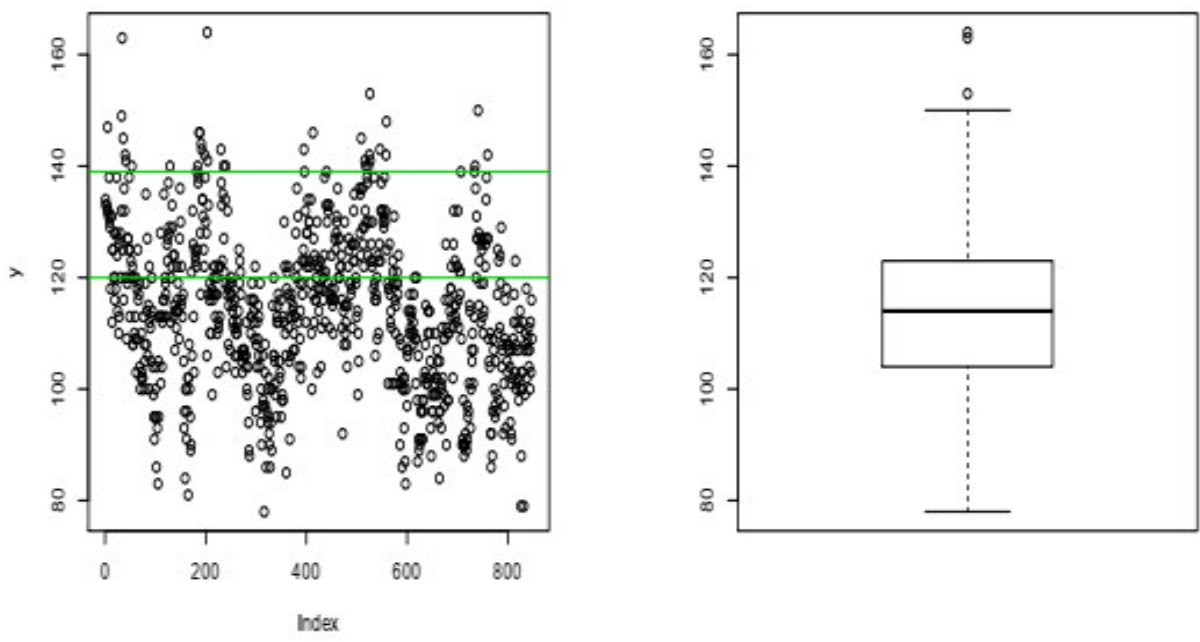

Fig. 1: Plot of real systolic blood pressure data. Scatter plot (left) and boxplot (right). 

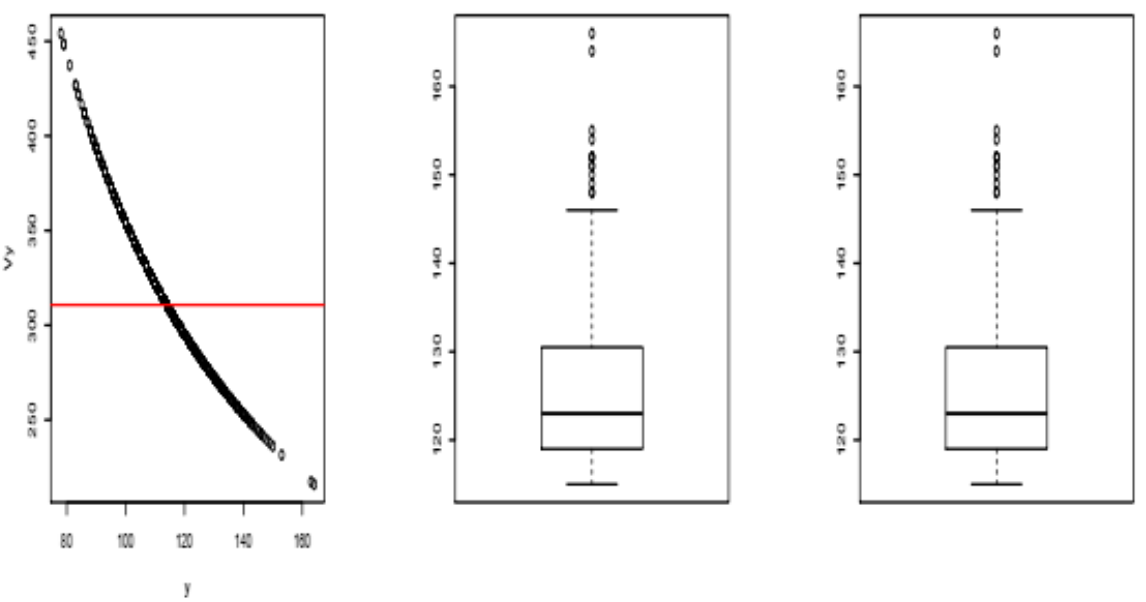

Fig. 2: Appropriate data $y^{*}$ learning by schemes by SFPDF and DFPDF schemes.

The utility of the statistic, $T(y)$, in the normal systolic blood detection process is shown in Figure 3 . The red line denotes the natural threshold. It can be seen that positive values of $T(y)$ naturally correspond to likely normal systolic blood pressure measurements. Furthermore, there exist differences in the normal SBP mining capabilities of the methods. It follows that, the narrower the curve, the better the detection, with both SFPDF and DFPDF able to learn physiology much better than DPDF, leading to suitable data driven normal values of SBP. The potential of the proposed methods for learning physiology specific normal systolic blood pressure observations from training data is shown in Table II. Clearly, SFPDF and DFPDF exhibits the same performance and both outperform DPDF. Also, we are able to detect $98 \%$ of the normal systolic blood pressure measurements from the training dataset.
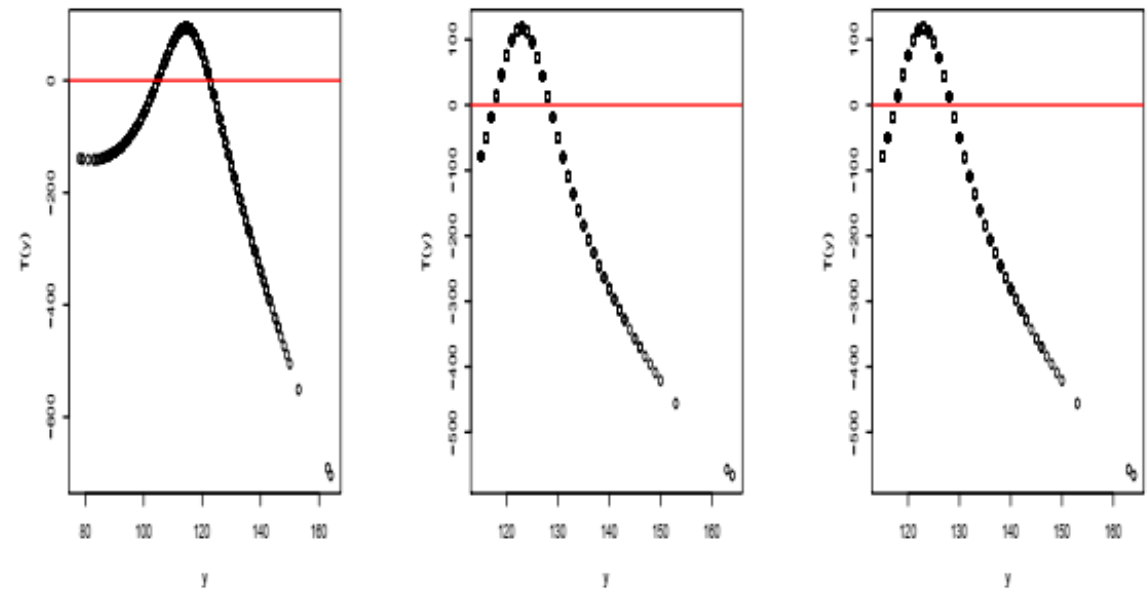

Fig. 3: The utility of the statistic $T(y)$ in DPDF (left), SFPDF (middle) and DFPDF (rigth). 
TABLE II: Real data: Performance of DPDF, SFPDF, and DFPDF with $\alpha_{0}=0.52$.

\begin{tabular}{lllr}
\hline & \multicolumn{3}{l}{ Performance measure } \\
\cline { 2 - 4 }$\hat{\theta}^{2}=61.37$ & Sensitivity & Specificity & Accuracy \\
\hline Scheme & SS & SP & ACC \\
\hline DPDF & 0.53 & 0.53 & 0.53 \\
SFPDF & 0.93 & 1.00 & 0.98 \\
DFPDF & 0.93 & 1.00 & 0.98 \\
\hline
\end{tabular}

\subsection{Example 2: Perturbed systolic blood pressure data}

We consider a second example in which the true systolic blood pressure data were perturbed randomly by an additive noise at the level of variability of the real dataset. In particular, the simulation followed the data generative process of the form

$$
\tilde{y}_{i}=y_{i}+\varepsilon_{i}, \quad \varepsilon_{i} \sim N\left(0, \sigma_{\varepsilon}^{2}\right),
$$

where $y_{i}$ denotes the original systolic blood pressure measurement and $\sigma_{\varepsilon}^{2}$ is set as the variance of the original dataset. The nature of the synthetic data is shown in Figure 4. The appropriateness of the dataset for normality pattern learning is evident. The results obtained from the application of the developed schemes on $\tilde{y}$, are not very different from those obtained in Example 1.

Figure 5 illustrates the automated training data preprocessing potential of SFPDF and DFPDF in terms of the acquisition of $y^{*}$. Again, the use of the statistic, $V(y)$, allows appropriate selection of subset of training data for efficient SBP normal pattern detection. Also, both schemes yield the same subset.

The underlying characteristics of the proposed statistic, $T(y)$, and its detection potential for the simulated systolic blood pressure data is demonstrated in Figure 6. The intrinsic differences among the three schemes are clearly seen.

Table III shows the sensitivity, specificity and accuracy of the three schemes for the simulated dataset considered. Again, it is easily seen that both SFPDF and DFPDF outperform DPDF by an order of magnitude. However, slight performance improvement is recorded for SFPDF over DFPDF in terms of specificity and accuracy.
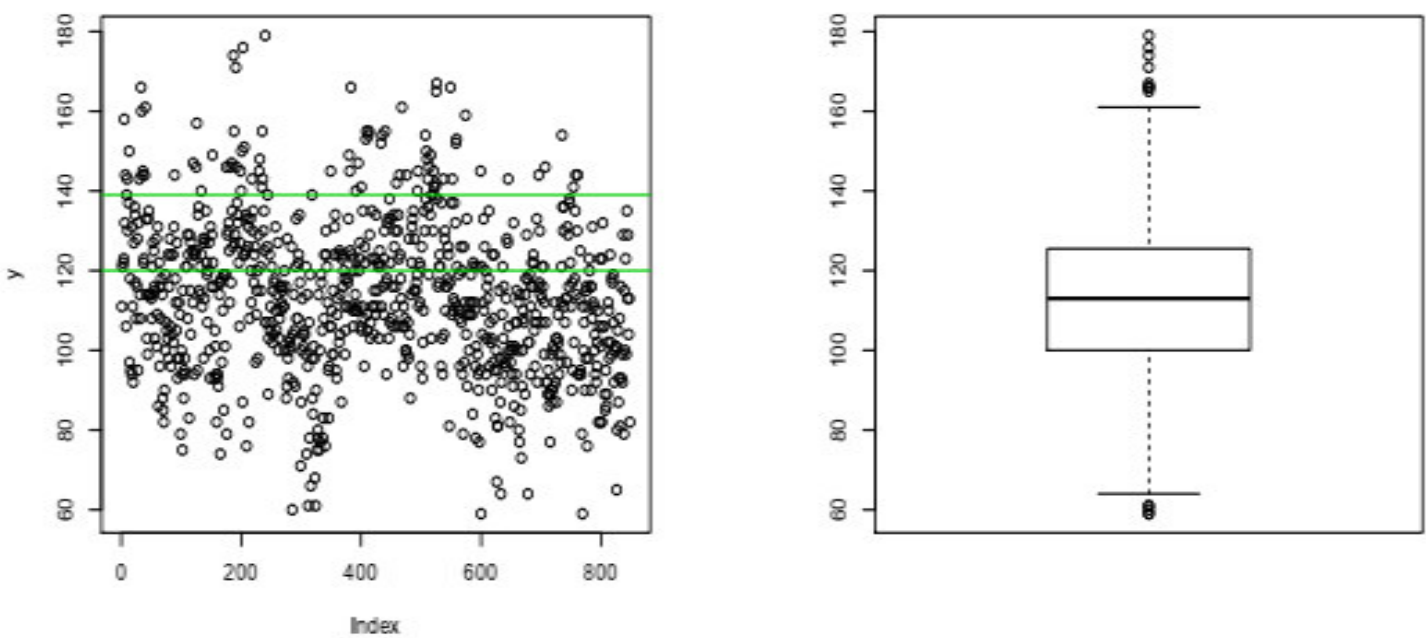

Fig. 4: Plot of simulated systolic blood pressure measure. Scatter plot (left) and boxplot (right). 

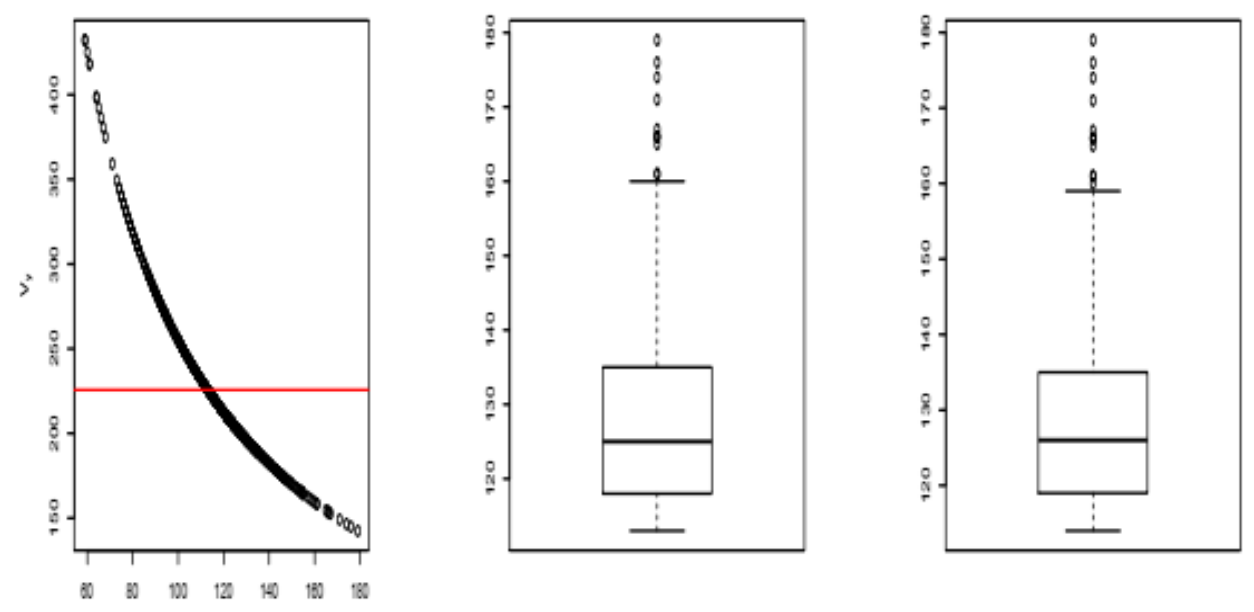

Fig. 5: Perturbed SBP data: Nature of subset data, $y^{*}$, obtained with SFPDF and DFPDF schemes.
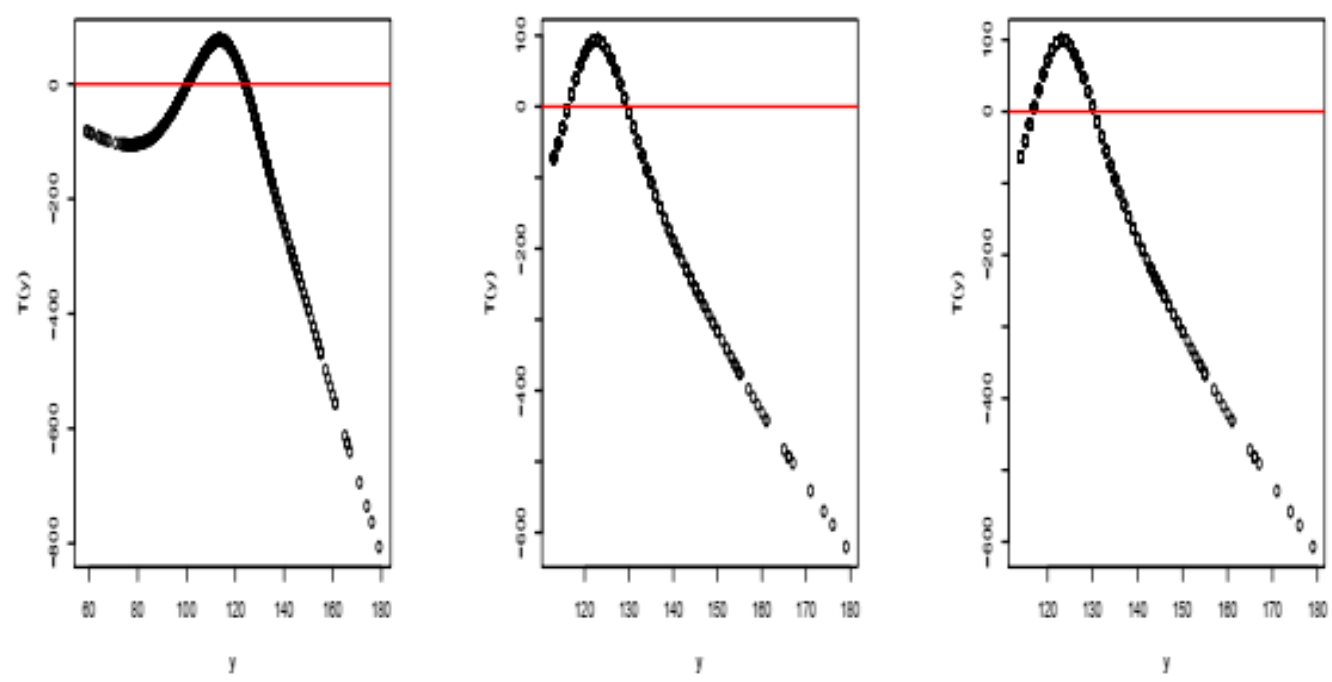

Fig. 6: Perturbed data: Nature of statistic, $T(y)$, with DPDF (left), SFPDF (middle) and DFPDF (rigth). 


\section{TABLE III: Performance of DPDF, SFPDF and DFPDF using perturbed systolic blood pressure data with $\alpha_{0}=0.52$.}

\begin{tabular}{lllr}
\hline & \multicolumn{3}{c}{ Performance measure } \\
\cline { 2 - 4 }$\hat{\theta}^{2}=56.56$ & Sensitivity & Specificity & Accuracy \\
\hline Scheme & SS & SP & ACC \\
\hline DPDF & 0.65 & 0.57 & 0.59 \\
SFPDF & 1.00 & 0.98 & 0.98 \\
DFPDF & 1.00 & 0.96 & 0.97 \\
\hline
\end{tabular}

\subsection{Example 3: Simulation}

In this simulation, 1000 synthetic systolic blood pressure observations were generated based on the sample mean and variance of the original dataset, $y$, such that the vital features of the real data are somewhat preserved. The sampling distribution of the synthetic data followed normal

$$
y_{i} \sim N\left(\mu_{y}, \sigma_{y}^{2}\right), i=1,2, \ldots, n,
$$

where $y$ denotes the original systolic blood pressure dataset and $\mu_{y}$ and $\sigma_{y}^{2}$ are its mean and variance respectively. The estimated smoothing parameter for this dataset is $\hat{\theta}^{2}=60.37$. Let $y$ denotes the vector of synthetic blood pressure measurement. We apply the developed schemes using $y$ based on an $\alpha_{0}$ value as in Examples 1 and 2 considered in subsection 4.1 and subsection 4.2 respectively. Figure 7 shows the plot of the data.

Figure 8 shows the distribution of the subset, $y^{*}$, of the simulated dataset, $y$ considered appropriate by SFPDF and DFPDF. Figure 9 illustrates the nature of the three schemes in terms of the statistic, $T(y)$, and Table IV gives their corresponding detection performances. DFPDF exhibits marginal improvement over its SFPDF counterpart.
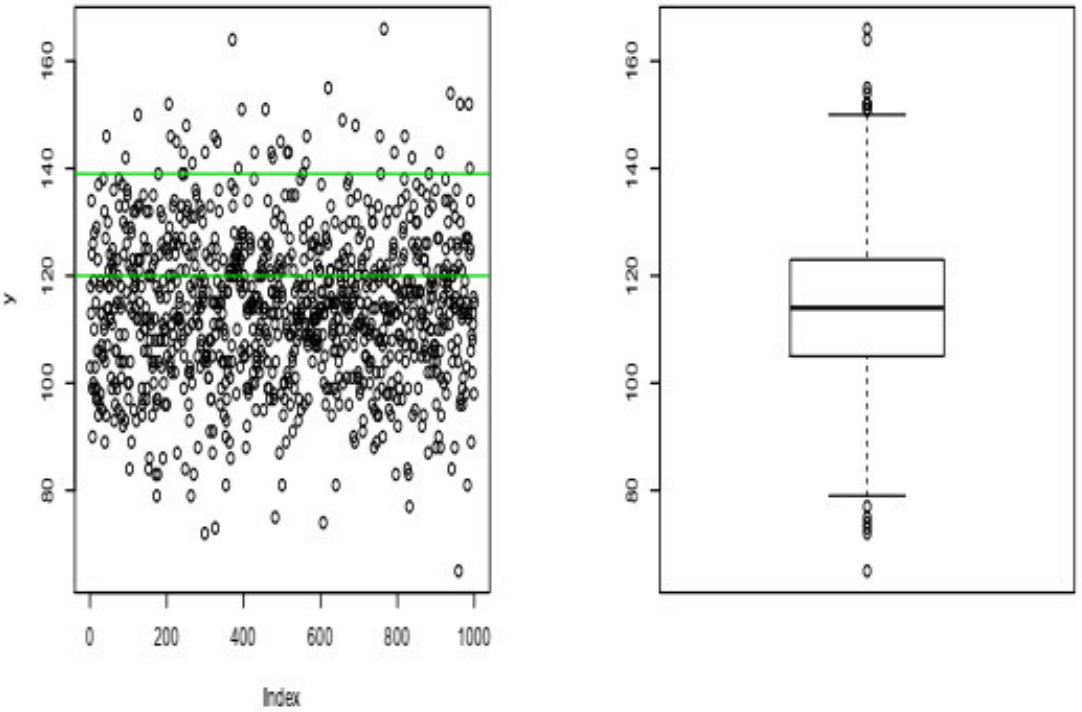

Fig. 7: Plot of simulated systolic blood pressure measure data. Scatter plot (left) and boxplot (right). 

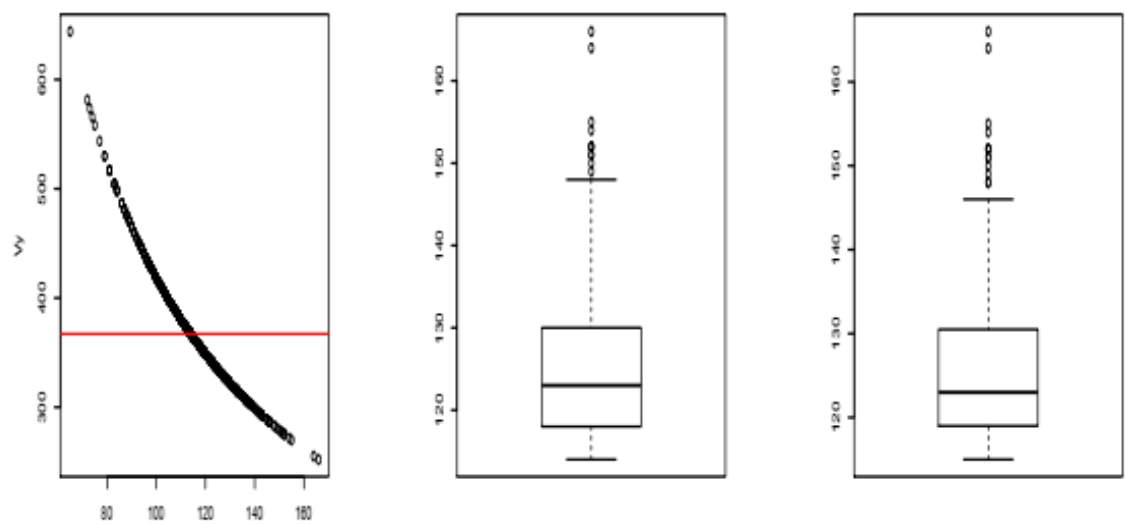

Fig. 8: Perturbed SBP data: Nature of appropriate data, $y^{*}$, obtained with SFPDF and DFPDF schemes.
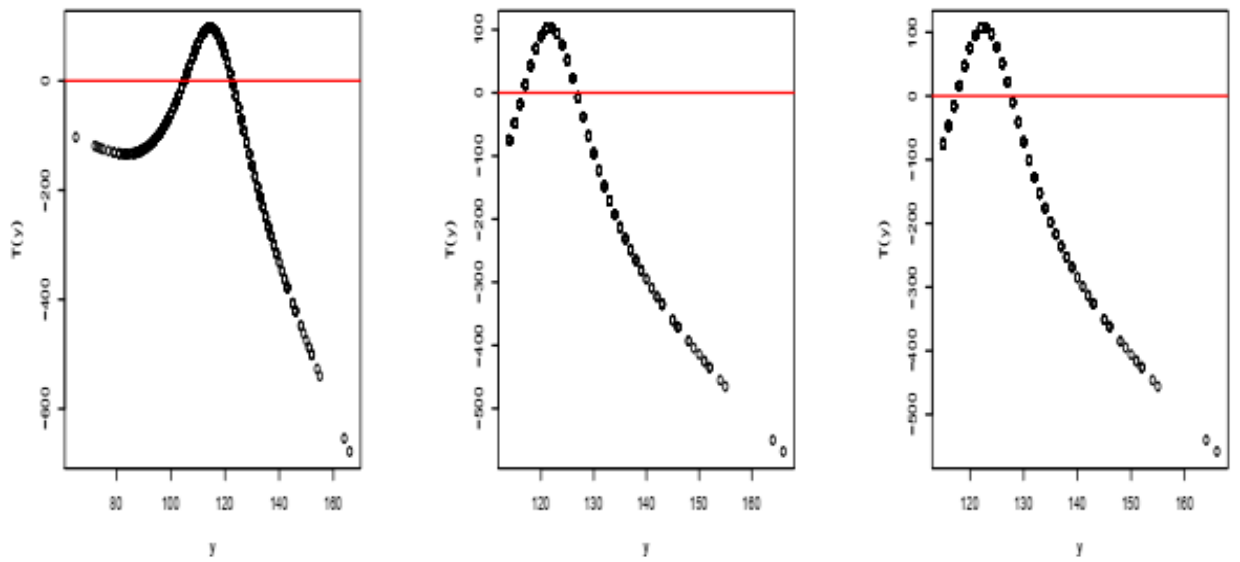

Fig. 9: Perturbed data: Nature of statistic, $T(y)$ with DPDF (left), SFPDF (middle) and DFPDF (rigth).

TABLE IV: Performance of DPDF, SFPDF and DFPDF based on simulated systolic blood pressure data with $\alpha_{0}=0.52$.

\begin{tabular}{lllr}
\hline & \multicolumn{3}{c}{ Performance measure } \\
\cline { 2 - 4 }$\hat{\theta}^{2}=60.37$ & Sensitivity & Specificity & Accuracy \\
\hline Scheme & SS & SP & ACC \\
\hline DPDF & 0.53 & 0.54 & 0.54 \\
SFPDF & 0.87 & 0.96 & 0.94 \\
DFPDF & 0.90 & 1.00 & 0.98 \\
\hline
\end{tabular}

\section{Conclusion}

In this paper, we have presented novel non-parametric statistical approaches for automated normal systolic blood pressure detection from training data, based on statistics derived from the probability density function (pdf) of the 
training data in the absence of known normal pattern. The pdf of the given training data is estimated via kernel density estimation and utilized to obtain an appropriate data from the training data, based on robust statistics of either its pdf or the data itself. Furthermore, incorporation of appropriate data pre-processing modules into developed artificial intelligence (AI) improves detection performance. In particular, the real data application recorded performance improvement of magnitude, 35\% in sensitivity for both SFPDF and DFPDF, $41 \%$ in specificity for SFPDF and 39\% for DFPDF. In terms of accuracy, SFPDF recorded a 39\% improvement while DFPDF registered 38\%. The perturbed data application also registered substantial improvements in performance across sensitivity $(40 \%)$, specificity $(47 \%)$ and accuracy $(45 \%)$. The proposed methods have the potential of transforming healthcare by building robust automated systolic blood pressure monitoring system for pragmatic and timely monitoring, if integrated into prototype blood pressure devices utilized in existing blood pressure monitoring systems, especially those involved in mobile health monitoring. Thus, making healthcare more accessible, through reduced cost, reduced health insurance claims and allowing remote monitoring by physicians any time anywhere.

\section{ACKNOWLEDGMENT}

The authors would like to register their indebtedness to the Government of Ghana for supporting this research through the teaching and research grant and Biofourmis Private Company Limited, Singapore for the provision of research data.

\section{REFERENCES}

Bishop, C. M. (1994). Novelty detection and neural network validation. IEE Proceedings-Vision, Image and Signal processing, 141(4), 217-222.

Buist, M. D., Burton, P. R., Bernard, S. A., Waxman, B. P. \& Anderson, J. (1999). Recognizing clinical instability in hospital patients before cardiac arrest or unplanned admission to intensive care: A pilot study in a tertiarycare hospital. Medical Journal of Australia, 171 (1), 22-25.

Centre for Clinical Practice at NICE (UK. (2007). Acutely ill patients in hospital: recognition of and response to acute illness in adults in hospital.

Duckitt, R., Buxton-Thomas, R., Walker, J., Cheek, E., Bewick, V., Venn, R., \& Forni, L. (2007). Worthing physiological scoring system: derivation and validation of a physiological early-warning system for medical admissions. An observational, population-based single-centre study. British journal of anaesthesia, 98 (6), 769-774.

Evans, D., Hodgkinson, B. \& Berry, J. (2001). Vital signs in hospital patients: a systematic review. International journal of nursing studies, 38 (6), 643-650.

Gao, H., McDonnell, A., Harrison, D. A., Moore, T., Adam, S., Daly, K., Esmonde, L., Goldhill, D. R., Parry, G. J. \& Rashidian, A. (2007). Systematic review and evaluation of physiological track and trigger warning systems for identifying at-risk patients on the ward. Intensive care medicine, 33 (4), 667-679.

Gardner-Thorpe, J., Love, N., Wrightson, J, Walsh, S. \& Keeling, N. (2006). The value of modified early warning score (mews) in surgical inpatients: a prospective observational study. The Annals of The Royal College of Surgeons of England, 88 (6), 571-575.

Khalid, S., Clifton, D. A., \& Tarassenko, L. (2013). A Bayesian patient based model for detecting deterioration in vital signs using manual observations. In International Symposium on Foundations of Health Informatics Engineering and Systems. Springer, 146-158.

Markou, M., \& Singh, S. (2003). Novelty detection: a review_part 1: statistical approaches. Signal processing, 83 (12), 2481-2497.

Markou, M., \& Singh, S. (2003). Novelty detection: a review_part 2:: neural network based approaches. Signal processing, 83 (12), 2499-2521.

Mensah, D. K., Assabil, S. \& Eyiah-Bediako, F. (2019). Data-driven approach to smoothing parameter estimation in univariate physiological vital signs kernel density estimation. 12, 2019, unpublished manuscript.

Pimentel, M. A., Clifton, D. A., Clifton, L., Watkinson, P. J., \& Tarassenko, L. (2013). Modelling physiological deterioration in post-operative patient vital-sign data. Medical \& biological engineering \& computing, 51(8), 869-877.

Vihinen, M. (2012, June). How to evaluate performance of prediction methods? Measures and their interpretation in variation effect analysis. In BMC genomics (Vol. 13, No. 4, p. S2). BioMed Central. 